\title{
Ortodontia baseada em evidência científica: incorporando ciência na prática clínica
}

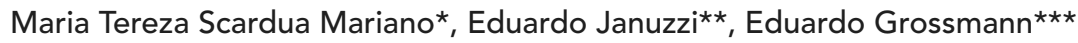

\begin{abstract}
Resumo
O objetivo deste artigo é despertar o ortodontista e conscientizá-lo sobre a importância da tomada de decisão baseada em evidência científica no cuidado aos pacientes. Serão descritos os passos essenciais para a prática da Odontologia baseada em evidência (OBE), assim como os princípios da ciência e da pesquisa. Existem caminhos adequados para a busca da informação de qualidade, sendo esses a única garantia de encontrar artigos válidos. Na seleção de artigos científicos, o primeiro passo é definir o seu desenho, pois para cada dúvida clínica há um delineamento adequado capaz de respondê-la. Dessa maneira, questões sobre tratamento, etiologia, diagnóstico, prognóstico ou prevenção só podem ser respondidas por um artigo que tenha sido delineado para tal. O conhecimento da alocação randomizada, do mascaramento e do grupo-controle é fundamental para que possamos realizar uma leitura crítica dos artigos científicos, reconhecendo os que merecem credibilidade. Em meio a tantas publicações, precisamos definir, com segurança, o que deve ser incorporado ao nosso conhecimento e o que deve ser incorporado à prática clínica, mudando a nossa conduta. Desse modo, poderemos oferecer aos nossos pacientes opções terapêuticas mais consistentes e previsíveis.
\end{abstract}

Palavras-chave: Ortodontia baseada em evidência. Projeto de pesquisa epidemiológica.

\section{INTRODUÇÃO}

O início da década de 90 introduziu a tomada de decisão baseada em evidência para os cuidados em saúde ${ }^{19}$. A Odontologia baseada em evidência (OBE) é o uso consciencioso, explícito e judicioso da melhor evidência disponível para a tomada de decisão sobre os cuidados de cada paciente ${ }^{2}$. Essa nova prática da Odontologia integra a informação científica de alta qualidade, advinda de pesquisas científicas com a experiência clínica e os valores de cada paciente.
Tragicamente, alguns profissionais da saúde negam a validade e a utilidade da ciência e o paradigma da evidência científica. Em vez de defenderem argumentos alicerçados na ciência, eles aceitam afirmações dogmáticas baseadas no empirismo, talvez por se sentirem ameaçados em suas autoridades, em suas limitações e interesses. Em meados da década de 80 , Peter Vig ${ }^{23}$ relatou que os ortodontistas davam maior ênfase ao aprimoramento da arte do que ao aprimoramento da ciência. O fato é que, ainda hoje, deparamo-nos com uma Odontologia

* Mestre em Disfunção Temporomandibular - UNIFESP. Especialista em Ortodontia - USP/Bauru. Especialista em Saúde Baseada em Evidência Científica - Hospital Sírio Libanês. Diplomada pelo Board Brasileiro de Ortodontia.

** Mestre em Disfunção Temporomandibular - UNIFESP. Especialista em Prótese e Periodontia USP/Bauru.

*** Professor doutor da Universidade Federal do Rio Grande do Sul (UFRGS). Diretor do Centro de Dor e Deformidade Orofacial (CENDDOR), Porto Alegre/RS. 
que se fundamenta nas experiências pessoais, na autoridade de indivíduos com maiores títulos acadêmicos, em ministradores de cursos, em autores de livros e em teorias fisiopatológicas ${ }^{3}$. O problema de se basear no conhecimento adquirido por meio de experiência pessoal (empirismo) é que a percepção humana é afetada por fatores tais como experiências passadas, crença na expectativa de sucesso, erros predeterminados, motivação, distorção de memória, possibilidade de remissão espontânea e a natureza cíclica de determinadas doenças, como as disfunções temporomandibulares (DTM) e outras. Por outro lado, a ciência é considerada um método superior para adquirir conhecimento, por ser desprovida de crenças, valores e emoções pessoais ${ }^{9}$.

Muitos conceitos em Ortodontia têm sido publicados como fatos, quando na verdade são teorias e hipóteses não testadas. Embora esses conceitos parecessem lógicos, foram derrubados por pesquisas científicas válidas. Em 1985, Proffit ${ }^{18}$ citou vários exemplos: terapia miofuncional, aparelhos funcionais para adultos e corticotomia para acelerar o alinhamento dentário. Além desses, um outro conceito em Ortodontia professou por décadas que um componente anterior de força irruptiva do terceiro molar causava apinhamento dos incisivos. Em um artigo publicado em 1972, Björk e Skieller ${ }^{4}$ já relatavam o resultado de um estudo prospectivo em que os pacientes foram acompanhados durante 6 anos e não se encontrou evidência de que o terceiro molar fosse fator de risco para o apinhamento dos incisivos. Mais recentemente, um ensaio clínico randomizado foi realizado e confirmou não haver associação entre apinhamento e a presença dos terceiros molares ${ }^{13}$.

Revisões sistemáticas também não têm sustentado a teoria de que aparelhos funcionais podem aumentar o crescimento efetivo da mandíbulala, ${ }^{1,6,8,21}$. A OBE não contraindica o uso de aparelhos funcionais, mas desperta para os reais mecanismos responsáveis pela correção anteroposterior da má oclusão de Classe II, concluindo que existem mais efeitos dentários que esqueléticos e mais efeitos na maxila do que na mandíbula, sendo provável que ocorram muitas remodelações.

A prática da $\mathrm{OBE}$, portanto, tira a ênfase da prática da Odontologia baseada na experiência e na intuição pessoal, e dá especial atenção ao desenho das pesquisas, ao seu gerenciamento e à análise estatística ${ }^{3}$. Seu objetivo é ajudar os clínicos a realizar tratamentos mais efetivos, mais eficientes e mais previsíveis, fundamentando as tomadas de decisão sobre as condutas com os pacientes em provas científicas rigorosas ${ }^{15}$.

$\mathrm{Na}$ atual era da Odontologia baseada em evidências, os ortodontistas necessitam entender mais claramente os princípios da ciência e da pesquisa. O objetivo deste artigo é clarear o entendimento dos princípios da pesquisa científica, despertando os ortodontistas para a importância de pesquisas clínicas ortodônticas bem construídas e sua aplicação na tomada de decisão clínica com os pacientes.

\section{TIPOS DE ESTUDO}

A quantidade de artigos disponíveis em Odontologia é vasta e crescente, gerando um excesso de informações que precisam ser selecionadas. A avaliação crítica da literatura é essencial para que se defina o que será incorporado ao nosso conhecimento e o que mudará nossa conduta clínica.

O primeiro passo para a avaliação crítica é definir o tipo do estudo, pois cada desenho de estudo é adequado para responder a uma determinada questão. Dessa maneira, apenas a leitura dos resumos de muitos artigos será suficiente para descartá-los, se não apresentarem um desenho adequado para responderem ao que se propõem (Fig. 1).

Os artigos científicos são classificados de acordo com a metodologia empregada, sendo importante que o ortodontista conheça as características e finalidades de cada um deles.

Por exemplo, o estudo que responde a questões relacionadas à história natural de uma doença, ao seu prognóstico ou identifica fatores etiológicos é o estudo coorte. Nele, um grupo de pessoas é exposto a um fator de risco e outro grupo de pessoas não. 


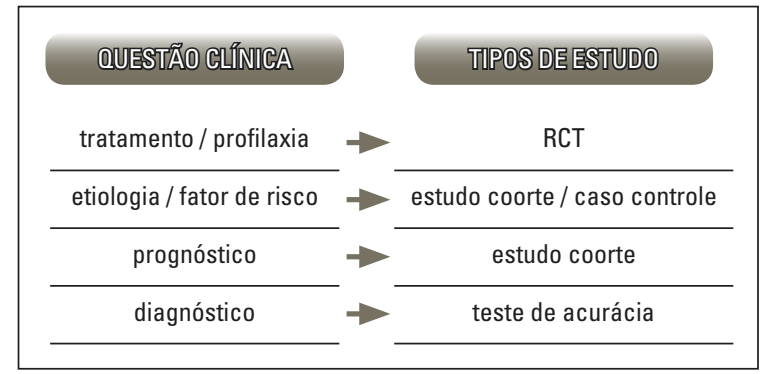

FIGURA 1 - Delineamento do estudo de acordo com a questão clínica.

Tais grupos são apenas observados ao longo do tempo, para se identificar qual deles experimenta o desfecho clínico de interesse ${ }^{11}$. Assim, poderíamos seguir um grupo de pacientes que apresentasse mordida aberta (exposto ao fator de risco) e outro grupo com ausência de mordida aberta (não exposto), mas todos com ausência de sinais e sintomas de DTM. Após um período adequado de tempo, poderíamos detectar se existe diferença entre o exposto e o não exposto, em relação à incidência de sinais ou de sintomas de DTM entre os grupos.

Em relação às decisões sobre terapêutica, só são aceitos resultados de pesquisa de estudos controlados, nos quais os pacientes foram escolhidos aleatoriamente para formar grupos (de tratamento e de controle). Cada um dos grupos receberá intervenção diferente, ou um grupo servirá como controle, não recebendo intervenção. Os ensaios clínicos randomizados (RCT) são descritos como "padrão ouro" na avaliação de questões terapêuticas em saúde ${ }^{24}$.

As revisões sistemáticas da literatura constituem um tipo de estudo que associa estudos semelhantes sobre um determinado tópico como se fizessem parte de um único, obtendo-se uma resultante do efeito terapêutico no conjunto ${ }^{3}$.

Revisões sistemáticas são diferentes das nossas históricas revisões narrativas. Nas primeiras, uma sistemática busca da literatura é realizada e, após uma criteriosa avaliação, os estudos considerados válidos serão incluídos na revisão. Por outro lado, nas revisões narrativas o autor inclui ou exclui alguns artigos, sem uma prévia busca sistemática e sem critérios seguros de avaliação. Dessa forma, por uma decisão arbitrária do autor, alguns estudos são incluídos na revisão narrativa da literatura ${ }^{15}$.

\section{ALOCAÇÃO RANDOMIZADA}

A alocação randomizada consiste em utilizar algum método que propicie a distribuição ao acaso dos pacientes de um estudo para cada grupo (controle / tratamento), tendo por objetivo a formação de dois grupos similares ${ }^{20}$. A utilização de um processo randômico, a fim de conceder aos participantes do estudo a mesma probabilidade de receber a intervenção ou de ser controle, revolucionou a prática clínica, ao ser usado pela primeira vez no final da década de $40^{16}$.

A maior importância da randomização se deve ao fato de possibilitar uma distribuição equivalente de variáveis nos dois grupos, principalmente das desconhecidas, produzindo um equilíbrio entre os diversos fatores de risco que poderiam influenciar no desfecho clínico a ser medido ${ }^{20}$.

\section{MASCARAMENTO}

Pacientes de um ensaio clínico podem mudar seu comportamento de um modo sistemático (isto é, tendencioso), se ficarem cientes da intervenção a que estão sendo submetidos. Uma maneira de minimizar esse efeito é mascarar (cegar) os participantes, na tentativa de que não reajam de maneira diferente, prejudicando a validade do estudo.

Os termos unicego, duplo cego e triplo cego algumas vezes são usados, mas o seu significado é ambíguo. O importante é descrever o que foi feito: se os pacientes estão cegos, se o clínico responsável pelo atendimento ou o pesquisador que fará a mensuração dos resultados estão cegos.

Em Ortodontia, normalmente só é possivel o mascaramento para o pesquisador responsável pela coleta dos dados, o que aumenta a validade do estudo, pois evita a tendenciosidade na mensuração dos resultados ${ }^{11}$. 


\section{A HIERARQUIA DA EVIDÊNCIA}

Nem toda evidência científica tem o mesmo peso. As revisões sistemáticas e os ensaios clínicos estão localizados no topo da hierarquia, enquanto os estudos animais e in vitro ocupam o mais baixo nível de evidência na tomada de decisão sobre tratamento $^{7}$ (Fig. 2). Entretanto, quando a dúvida clínica se refere a prognóstico ou a fatores de risco, os estudos coorte ocupariam o nível 1 de evidência.

Tão importante quanto a hierarquia da evidência é a qualidade metodológica dos estudos. Nesse sentido, faz-se necessária uma leitura crítica dos artigos científicos. Fica evidente que relatos, tais como experiência e testemunho do próprio clínico, são cientificamente antiéticos e não devem ser considerados.

\section{A BUSCA DA INFORMAÇÃO DE QUALIDADE}

Idealmente, a prática da Saúde Baseada em Evidências deve ser um processo sistematizado, que envolve algumas fases: formular uma questão clínica relevante, a partir da situação clínica do paciente; buscar na literatura artigos clínicos relevantes; analisar criticamente as evidências - provas científicas - em relação à sua validade e aplicabilidade ${ }^{3}$; e, por fim, transferir as descobertas importantes para

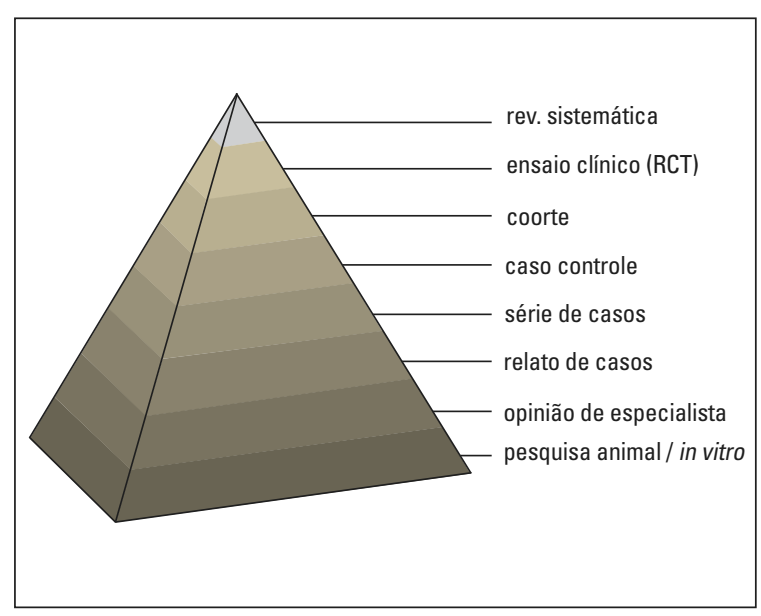

FIGURA 2 - Níveis de evidência para tomada de decisão sobre tratamento?. a prática clínica (Fig. 3).

A formulação da pergunta, que se origina de uma dúvida no atendimento ao paciente, serve como bússola, norteando a busca da resposta apropriada. Na sequência, é necessário classificá-la: pergunta sobre tratamento, pergunta sobre etiologia, pergunta sobre diagnóstico ou pergunta sobre prevenção ${ }^{3,5}$.

Existem vários caminhos para a busca da informação na Odontologia, tais como: consultar um livro, procurar numa coleção particular de artigos ou consultar um especialista ${ }^{14}$. Consultar um livro sempre implica em risco de se obter informação desatualizada - um livro leva de 1 a 2 anos entre a redação e a publicação.

Procurar na própria coleção de artigos não garante que as evidências sejam as melhores disponíveis. Consultar um especialista é correr o risco da tendenciosidade - ele pode oferecer não o que existe de melhor, mas o que ele pensa ser melhor.

A forma correta de buscar a informação de qualidade é a pesquisa on line de artigos científcos. Para uma busca efetiva, é necessário entender como funcionam as bases de dados, identificando seus recursos. A Medline é a maior base de dados do mundo sobre pesquisas em saúde. O site PubMed (www.pubmed.org) é o local onde a base de

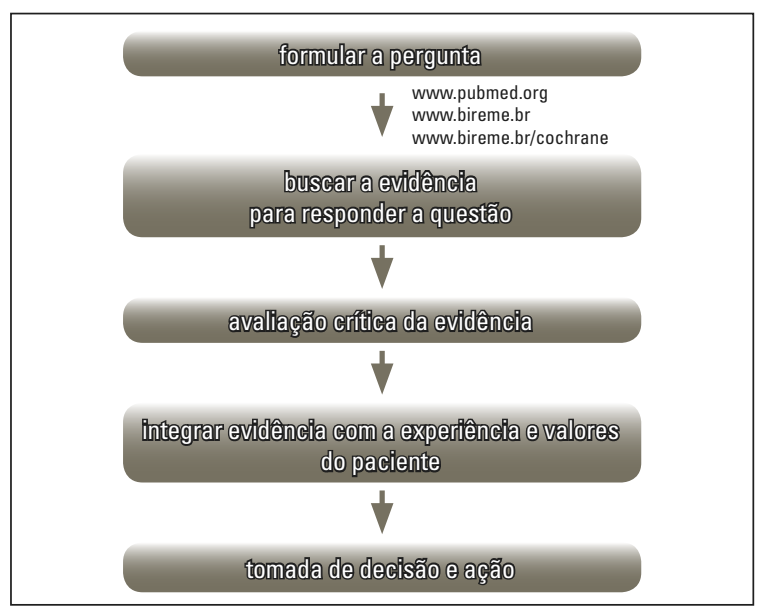

FIGURA 3 - Passos essenciais para prática da OBE. 
dados Medline pode ser acessada gratuitamente.

A Lilacs (Literatura Latino-Americana e do Caribe em Ciências da Saúde) é uma base de dados cooperativa do Sistema Bireme, que compreende a literatura relativa às Ciências da Saúde publicada nos países da região a partir de 1982. Está disponível em inglês, português e espanhol. O site da Bireme pode ser acessado gratuitamente pelo endereço www.bireme.br.

A Cochrane Library (www.bireme.br/cochrane) é a biblioteca virtual considerada como uma das melhores fontes de evidência em saúde, pois nela somente são indexados ensaios clínicos controlados randomizados e revisões sistemáticas, enquanto nas outras bases de dados é necessária a utilização de estratégias de busca para selecionarmos o tipo de estudo desejado. $\mathrm{O}$ acesso à Cochrane também é gratuito.

\section{FATORES DE CONFUSÃO (VIÉS DE CONFUSÃO)}

Um dos objetivos da pesquisa em modelo experimental seguro é controlar (eliminar ou neutralizar) os fatores de confusão. Quando os fatores de confusão não são controlados, os efeitos do tratamento são mascarados pela influência de outros fatores, não podendo ser isolados nem analisados. Se os resultados desses estudos são aceitos, existe uma forte possibilidade de promoverem impacto negativo nos cuidados com os pacientes, isto é, os tratamentos necessários serão ignorados e os desnecessários adotados.

A utilização de pacientes humanos (em lugar de experimentos com animais, células ou tecidos) em pesquisas complica o controle das variáveis de confusão. Fatores psicológicos e cognitivos podem influenciar nos resultados do estudo e, por isso, devem ser considerados. Além desses, há outros tipos de erros sistemáticos, como os relacionados à seleção da amostra e à aferição dos dados que precisam ser controlados. Esses são considerados ameaça para a validade interna e externa do estudo, representando alguns dos mais importantes fatores que o leitor deve considerar quando avalia os resultados de uma pesquisa ${ }^{9,11}$.

\section{EFEITO PLACEBO}

O efeito placebo é a melhora da saúde, observável, mensurável ou sentida, não atribuída ao tratamento. Para combater efeitos placebos reversos, muitos pesquisadores usam um placebo no grupo-controle. Quando a pesquisa envolve um placebo, tanto o grupo experimental quanto o de controle recebem o tratamento, mas um dos tratamentos é falso. Desse modo, o placebo permite a prática e a apresentação de variáveis psicológicas no grupo controle, sem comprometer a validade do tratamento no grupo experimental. Procedimentos considerados placebo são "procedimentos simulação", que são muito similares às técnicas de tratamento, mas não objetivam fornecer tratamento efetivo. Os resultados de pesquisa que não usam placebo ou procedimento simulação devem ser questionados ${ }^{9}$. O uso de placebos também é adequado na mensuração dos resultados, quando o estudo é cego para o avaliador.

\section{GRUPO-CONTROLE}

Algumas pesquisas, como relatos de caso clínico, não utilizam grupo-controle. Por outro lado, quando controles são requeridos e usados, a validade e a confiança do estudo são aprimoradas. Incluindo um grupo-controle em pesquisas experimentais, o pesquisador pode assegurar que o tratamento ou o fator de risco é diretamente responsável pelos resultados obtidos. Tipicamente, o pesquisador apresentará a condição de tratamento para o grupo experimental, enquanto o grupo-controle não receberá tratamento ou receberá tratamento com método padrão. No estudo coorte, um grupo está exposto a um fator de risco e o outro não (grupo de comparação).

Dos anos 50 até os anos 70, foi amplamente divulgado que desarmonias oclusais, particularmente desvios da relação cêntrica, causavam DTM $^{17}$. Entretanto, esses estudos careciam de controle ou de grupo de comparação. Estudos posteriores, 
incorporando grupo-controle ou comparativo, revelaram que pacientes assintomáticos também apresentavam desvios equivalentes ${ }^{12}$.

\section{TAMANHO E PODER DA AMOSTRA}

Suponhamos que alguém esteja lendo um ensaio clínico que compara duas terapias. Ele quer saber se o número de pacientes (tamanho da amostra) no estudo é suficiente para descartar o acaso do resultado encontrado. Quantos pacientes seriam necessários para fazer uma comparação adequada dos efeitos do tratamento? A resposta depende de quatro características do estudo: a magnitude da diferença dos desfechos entre os grupos, o erro alfa, o erro beta e a natureza dos dados do estudo ${ }^{11}$.

$\mathrm{O}$ pesquisador define previamente (baseado na experiência, na literatura e/ou na relevância clínica) o "grau de diferença" entre os grupos que será considerado clinicamente significativo. Quanto menor a diferença a ser encontrada em resposta à terapia, mais pacientes serão necessários para detectá-la ${ }^{10}$.

Os pesquisadores realizam pesquisas para testar uma hipótese. Essa hipótese é chamada nula quando não existe diferença na mensuração dos resultados entre os grupos tratados ou expostos. Por exemplo, o ensaio clínico ${ }^{22}$ que testou a hipótese de que a resposta da maxila à tração seria mais efetiva se realizada a expansão prévia da maxila, teve a hipótese nula com resultado. Ou seja, não houve diferença na magnitude do avanço maxilar (desfecho) entre os grupos com e sem a prévia expansão da maxila.

É preciso também considerar que as diferenças do efeito do tratamento entre os grupos podem ocorrer somente pelo acaso ${ }^{2}$. A probabilidade de ocorrer esse erro é representada pelo valor de "p", que varia de 0 a 1 . Normalmente, é estipulado um valor de 0,05 , o que significa que existe $5 \%$ de chance de mostrar uma diferença pelo mero acaso, e não porque ela realmente exista. Outra forma de se expressar seria dizer que um "p" de 0,05 significa que a cada 20 desfechos medidos 1 foi meramente pelo acaso $^{9,10,11}$. A conclusão em um estudo de que há diferença entre as hipóteses testadas que, na verdade, não existe, é chamada erro alfa ou erro tipo I.

O erro tipo II, ou erro beta, é a probabilidade de um estudo não encontrar diferenças entre os grupos estudados, quando na verdade elas existem. Frequentemente, é estabelecido em 0,20, ou seja, é considerado que há $20 \%$ de chance de o estudo omitir verdadeiras diferenças nos resultados dos grupos estudados.

A fim de evitar esses erros, atenção especial deve ser prestada à representatividade da amostra. Além disso, quanto maior o tamanho da amostra maior a probabilidade de que ela represente as características da população geral, proporcionando maior generalização dos resultados do estudo.

Finalmente, deve-se considerar o desvio-padrão (d.p.) esperado, ou seja, a dispersão dos dados de interesse ao redor da média. Quanto maior for a variação entre os pacientes, com relação à característica que está sendo medida, mais difícil será confiar que as diferenças observadas (ou a ausência de diferenças) entre os grupos não ocorram por causa dessa variação e sim por uma diferença real entre os efeitos do tratamento. Em outras palavras, quanto maior a variação entre os indivíduos menor será o poder estatístico ${ }^{2,9,10,11}$.

\section{CONCLUSÕES}

Informações baseadas em provas científicas são as mais confiáveis para uma tomada de decisão conscienciosa em relação à abordagem clínica oferecida aos pacientes. Diante das inúmeras publicações e da crescente inovação tecnológica, torna-se necessário que o ortodontista se familiarize com a utilização das ferramentas para a prática da Odontologia Baseada em Evidência. Pacientes ortodônticos merecem um alto nível de cuidado, que somente é alcançado por meio do uso judicioso das melhores informações disponíveis. Assim, o profissional deve estar apto a realizar uma leitura crítica dos artigos científicos, decidindo, com segurança, qual conhecimento será incorporado à sua prática clínica.

Enviado em: outubro de 2006 Revisado e aceito: julho de 2007 


\title{
Scientific evidence-based Orthodontics: incorporating science within clinic practice
}

\begin{abstract}
The aim of this article is to warn the orthodontist about the importance of making decision based on scientific evidence when taking care of the patients. It describes the main steps for the Dentistry practices based in evidence (EBD) as well as the science and research principles. There are adequate ways for the search of quality information. While selecting the scientific articles, the first step would be defining its design, since for each question there is an adequate delineation able to answer it. Questions about treatment, etiology, diagnostic, prognostic or prevention could be answered in the article if it has been delineated for that. Understanding the meaning of randomized allocation, blinding and control group is essential for a critical reading; selecting those articles that deserve credibility. Among so many publications one needs to identify precisely about what must be incorporated to his knowledge as well as to the clinical practices.
\end{abstract}

Keywords: Evidence-based Orthodontics. Epidemiologic research design.

\section{REFERÊNCIAS}

1. AELBERS, C. M.; DERMAUT, L. R. Orthopedics in Orthodontics. Part I: fiction or reality: a review of the literature. Am. J. Orthod. Dentofacial Orthop., St. Louis, v. 110, no. 5, p. 513-519, 1996.

2. ATALLAH, A. N. Estrutura mínima do projeto de pesquisa clínica. In: ATALLAH, A. N. (Ed.). Medicina baseada em evidências: fundamentos da pesquisa clínica. São Paulo: Lemos Editorial, 1998. p. 59-65.

3. ATALLAH, A. N.; CASTRO, A. A. Medicina baseada em evidências: o elo entre a boa ciência e a prática clínica. Rev. Imagem, [s.I.], v. 20, n.1, p. 5-9, 1998. Disponível em <http://www.wpm.br/cochrane/ebm.htm>. Acesso em: 20 mar. 2009.

4. BJORK, A.; SKIELLER, V. Facial development and tooth eruption: an implant study at the age of puberty. Am. J. Orthod., St. Louis, v. 62, no. 4, p. 339-383, 1972.

5. CASTRO, A. A. Localizando informação para a prática clínica. In: ATALLAH, A. N. (Ed.). Medicina baseada em evidências: fundamentos da pesquisa clínica. São Paulo: Lemos Editorial, 1998. p. 78-92

6. CHEN, J. Y.; WILL, L. A.; NIEDERMAN, R. Analysis of efficacy of functional appliances on mandibular growth. Am. J. Orthod.

Dentofacial Orthop. St. Louis, v. 122, no. 5, p. 470-476, 2002

7. COOK, D. J.; GUYATT, G. H.; LAUPACIS, A.; SACKETT, D. L.; GOLDBERG, R. J. Clinical recommendations using levels of evidence for antithrombotic agents. Chest, Chicago, v. 108 p. 227S-230S, 1995. Supplement, 4.

8. DERMAUT, L. R.; AELBERS, C. M. Orthopedics in Orthodontics: fiction or reality: a review of the literature. Part II. Am. J. Orthod. Dentofacial Orthop., St. Louis, v. 110, no. 6, p. 667-671, 1996

9. DONALD, J. R.; EMILY, M. S.; DANIEL, J. R.; DARA, L. R. Understanding science and evidence-based decision making in Orthodontics. Am. J. Orthod. Dentofacial Orthop., St. Louis, v. 127, no. 5, p. 618-624, 2005.

10. DORIA FILHO, U. Introdução à bioestatística: para simples mortais. São Paulo: Elsevier, 1999.

11. FLETCHER, R. H.; FLETCHER, S. W. Epidemiologia clínica: elementos essenciais. 3. ed. Porto Alegre: Artmed, 1996.

12. FORSSELL, H.; KALSO, E. Application of principles of evidencebased Medicine to occlusal treatment for temporomandibular disorders: are there lessons to be learned? J. Orofac. Pain Carol Stream, v. 18, no. 1, p. 9-22, 23-32, 2004

13. HARRADINE, N. W.; PEARSON, M. H.; TOTH, B. The effect of extraction of third molars on late lower incisor crowding: a randomized controlled trial. Br. J. Orthod., London, v. 25, no. 2, p. 117-122,1998.
14. HAYNES, R. B.; McKIBBON, K. A.; FITZGERALD, D.; WALKER, C. J.; SACKETT, D. L. How to keep up with the medical literature: IV. Using the literature to solve clinical problems. Ann. Intern. Med., Philadelphia, v. 105, no. 4, p. 636- 640, 1986.

15. HUANG, G. J. Making the case for evidence-based Orthodontics. Am. J. Orthod. Dentofacial Orthop., St. Louis, v. 125, p. 405-406, 2004.

16. MEDICAL RESEARCH COUNCIL. Streptomycin treatment of pulmonary tuberculosis: a medical research Council investigation. BMJ, London, v. 2, p. 769-782,1948.

17. MOHL, N. Temporomandibular disorders: the role of occlusion, TMJ imaging, and electronic devices, a diagnostic update. J. Am. Coll. Dent., Bethesda, v. 58, p. 4-10, 1991.

18. PROFFIT, W. R. Who said that? Am. J. Orthod., St. Louis, v. 87, p. $166,1985$.

19. SACKETT, D.; ROSENBERG, W.; GRAY, J.; RICHARDSON, W. S. Evidence- based Medicine: what it is and what it isn't. BMJ, London, v. 312, p. 71- 72, 1996

20. SOARES, K. V. S.; CASTRO, A. A. Projeto de pesquisa para ensaio clínico randomizado. In: ATALLAH, A. N.; CASTRO, A. A. (Ed.). Medicina baseada em evidências: fundamentos da pesquisa clínica. São Paulo: Lemos Editorial, 1998. p. 66-77.

21. TULLOCH, J. F.; MEDLAND, W.; TUNCAY, O. C. Methods used to evaluate growth modification in Class malocclusion. Am. J. Orthod. Dentofacial Orthop., St. Louis, v. 98, no. 4, p. 340-347,1990.

22. VAUGHN, G. A.; MASON, B.; MOON, H. B.; TURLEY, P. K. The effects of maxillary protraction therapy with or without rapid palatal expansion: a prospective, randomized clinical trial. Am. J. Orthod. Dentofacial Orthop., St. Louis, v. 128, no. 3 , p. 299-309, 2005.

23. VIG, P. S. Reflections on the rationality of Orthodontics: toward a new paradigm. In: VIG, P. S.; ROBBINS, K. (Ed.). Science and clinical judgment in Orthodontics. Ann Arbor: Center for Human Growth an Development: University of Michigan, 1986. p. 15-60. Craniofacial Series, Monograph 19.

24. WORLD HEALTH ORGANIZATION. Council for International Organizations of Medical Sciences. International Ethical Guidelines for Biomedical Research Involving Human Subjects. Geneva: OPAS, 1996

\section{Endereço para correspondência}

Maria Tereza Scardua Mariano

Rua Chapot Presvot 100/801

CEP: 29.055-410 - Vitória / ES

E-mail: scardua@mariatereza.com.br 